\title{
The X-ray emission of the supernova remnant W49B observed with XMM-Newton
}

\author{
M. Miceli ${ }^{1,2,3}$, A. Decourchelle ${ }^{1}$, J. Ballet ${ }^{1}$, F. Bocchino ${ }^{3}$, J. P. Hughes ${ }^{4}$, U. Hwang ${ }^{5,6}$, and R. Petre ${ }^{6}$ \\ 1 DSM/DAPNIA/Service d'Astrophysique, AIM-UMR 7158, CEA Saclay, 91191 Gif-sur-Yvette Cedex, France \\ e-mail: miceli@astropa.unipa.it \\ 2 Dipartimento di Scienze Fisiche ed Astronomiche, Sezione di Astronomia, Università di Palermo, Piazza del Parlamento 1, \\ 90134 Palermo, Italy \\ 3 INAF - Osservatorio Astronomico di Palermo, Piazza del Parlamento 1, 90134 Palermo, Italy \\ 4 Department of Physics and Astronomy, Rutgers University, 136 Frelinghuysen Road, Piscataway, NJ 08854-8109, USA \\ 5 Center for Astrophysical Sciences, The Johns Hopkins University, 3400 Charles St., Baltimore MD 21218, USA \\ ${ }^{6}$ Laboratory for High Energy Astrophysics, Goddard Space Flight Center, Greenbelt, MD 20771, USA
}

Received 30 September 2005 / Accepted 24 February 2006

ABSTRACT

\begin{abstract}
Context. In the framework of the study of supernova remnants and their complex interaction with the interstellar medium, we report on an XMM-Newton EPIC observation of the Galactic supernova remnant W49B.

Aims. We investigated the spatial distribution of the chemical and physical properties of the plasma, so as to get important constraints on the physical scenario, on the dynamics of the supernova explosion, and on the interaction of the supernova remnant with the ambient interstellar clouds.

Methods. We present line images, equivalent width maps, and a spatially resolved spectral analysis of a set of homogeneous regions. Results. The X-ray spectrum of W49B is characterized by strong $\mathrm{K}$ emission lines from $\mathrm{Si}, \mathrm{S}, \mathrm{Ar}, \mathrm{Ca}$, and Fe. In all the regions studied, the X-ray spectrum is dominated by the emission from the ejecta and there is no indication of radial stratification of the elements. A high overabundance of $\mathrm{Ni}\left(\mathrm{Ni} / \mathrm{Ni}_{\odot}=10_{-1}^{+2}\right)$ is required in the bright central region and the previous detection of $\mathrm{Cr}$ and $\mathrm{Mn}$ line emission is confirmed. Spectra are described well by two thermal components in collisional ionization equilibrium. We observe spatial variations in the temperature, with the highest temperature found in the east and the lowest in the west.

Conclusions. Our results support a scenario in which the X-ray emission comes from ejecta interacting with a dense belt of ambient material, but another possibility is that the remnant is the result of an asymmetric bipolar explosion with the eastern jet being hotter and more Fe-rich than the western jet. The eastern jet is confined by interaction with ambient molecular clouds. Comparison of the observed abundances with yields for hypernova and supernova nucleosynthesis does not directly support the association of W49B with a $\gamma$-ray burst, although it remains possible.
\end{abstract}

Key words. X-rays: ISM - ISM: supernova remnants - ISM: individual object: W49B

\section{Introduction}

Supernova remnants (SNRs) are powerful sources of heavy elements and energy in the interstellar medium (ISM). The supernova supersonic material (ejecta) is expelled into the ambient medium and produces a shock front that heats and ionizes the ISM. As this shock expands into the ISM, a reverse shock associated with the deceleration of the ejecta propagates inward (in the reference frame of the expanding ejecta), thus heating the expelled material to X-ray emitting temperatures. The resulting $\mathrm{X}$-ray emission provides important information about the emitting conditions and the chemical composition of the ejecta.

The galactic supernova remnant W49B is one of the brightest ejecta-dominated SNRs observed in X-rays. It has a center-filled morphology in X-rays and a shell-like morphology in the radio band. According to Rho \& Petre (1998), W49B is similar to mixed-morphology supernova remnants, but its classification is uncertain. W49B is one of the 10 galactic sources with the highest surface brightness at $1 \mathrm{GHz}$ (Moffett \& Reynolds 1994). The diameter of the radio shell is $\sim 4^{\prime}$ and the distance to the remnant, estimated on the basis of HI absorption (Radhakrishnan et al. 1972), is $\sim 8 \mathrm{kpc}$ (considering the corrections by Moffett $\&$ Reynolds 1994).
In the X-ray band, W49B was first detected with the Einstein Observatory (Pye et al. 1984). The discovery of intense Fe XXV K line emission by EXOSAT (Smith et al. 1985) indicated that the X-ray emission of W49B is thermal and dominated by the ejecta. This result has been further confirmed by analysis of the ASCA spectrum (Fujimoto et al. 1995; Hwang et al. 2000). Ejecta-dominated remnants are expected to be relatively young, and while the age of W49B has not yet been determined accurately, published age estimates are in the range of 1000-4000 yr (Pye et al. 1984; Smith et al. 1985; Hwang et al. 2000). The images of $\mathrm{Si}, \mathrm{S}$, and $\mathrm{Fe} \mathrm{K}$ emission lines obtained with $A S C A$ indicate that the iron emission is confined to the inner part of the remnant, while silicon and sulfur emission appears to originate in the outer regions. This result has been interpreted as evidence of a stratified distribution of the elements (Fujimoto et al. 1995). The analysis of the same ASCA data carried out by Hwang et al. (2000) showed that broadband modeling of the remnant's global spectrum requires two thermal components and significant overabundances of $\mathrm{Si}, \mathrm{S}, \mathrm{Ar}, \mathrm{Ca}, \mathrm{Fe}$ with respect to the solar values. Recently, the comparison between data obtained in X-rays with the Chandra satellite and in the infrared with the Palomar observatory have revealed new details of the morphology of W49B (Keohane et al. 2004). In particular, the 
Table 1. Relevant information about the data.

\begin{tabular}{lcccc}
\hline \hline OBS_ID & CAMERA & $t_{\exp }(\mathrm{ks})^{*}$ & Mode & Filter \\
\hline 0084100401 & MOS1 & $18.6 / 15.8$ & Large Wind. & medium \\
0084100401 & MOS2 & $18.7 / 16.0$ & Large Wind. & medium \\
0084100401 & pn & $17.0 / 12.9$ & Full Frame & medium \\
0084100501 & MOS1 & $18.7 / 16.8$ & Large Wind. & medium \\
0084100501 & MOS2 & $18.7 / 16.3$ & Large Wind. & medium \\
0084100501 & pn & $17.0 / 14.6$ & Full Frame & medium \\
\hline
\end{tabular}

* Unscreened/Screened exposure time.

presence of an elongated structure in the $\mathrm{Fe} \mathrm{K}$ emission (interpreted by the authors as a jet), and the nondetection of a neutron star associated with the remnant, have been considered as making W49B consistent with theoretical predictions for a collapsar (e.g., MacFadyen \& Woosley 1999) and thus a candidate for a gamma-ray burst remnant.

Here we present the analysis of an XMM-Newton EPIC observation of W49B. The high sensitivity of XMM-Newton provides deep insights into the spatial distribution of the elements and the physical conditions in the remnant that lead to important constraints on the physical scenario. The paper is organized as follows: Sect. 2 presents the EPIC data and describes the data processing procedure; Sect. 3 presents the X-ray results in terms of the global spectrum (3.1), imaging (3.2), and spatially resolved spectral analysis (3.3). Section 4 discusses the results in the context of observations at other wavelengths and SN models. Finally, we draw our conclusions in Sect. 5.

\section{Data processing}

The XMM-Newton data presented here consist of two observations: Observation ID 0084100401 and 0084100501 (PI A. Decourchelle) performed on 3 April 2004 and 5 April 2004 with the EPIC MOS (Turner et al. 2001) and EPIC pn (Strüder et al. 2001) cameras, as summarized in Table 1. Both observations have the pointing coordinates $\alpha(2000)=19^{\mathrm{h}} 11^{\mathrm{m}} 13^{\mathrm{s}}$ and $\delta(2000)=9^{\circ} 7^{\prime} 1^{\prime \prime}$. A third data set 0084100601 (with the same pointing coordinates) is heavily contaminated by proton flares so was not used for our analysis.

The data were processed using the Science Analysis System (SAS V6.1.0). To create light curves, images, and spectra, we selected events with PATTERN $\leq 12$ for the MOS cameras, PATTERN $\leq 4$ for the pn camera, and FLAG $=0$ for both. To eliminate the contamination by soft proton flares, we screened the data by applying a count-rate limit on the light curves at high energies (10-12 keV for MOS and 12-14 keV for the pn camera). With a $\delta t=100 \mathrm{~s}$ binning for the light curves, we set the count-rate limit at 18 counts per bin for the MOS cameras, and 22 counts per bin for the pn camera. The exposure times are given in Table 1. For each camera we merged the screened event files of the two observations using the task MERGE.

All the images presented here are superpositions of the MOS1, MOS2, and pn images (obtained using the EMOS AIC task) and are background-subtracted, vignetting-corrected, and adaptively smoothed. Despite being relatively small in extent compared to the XMM-Newton field of view, W49B has a high X-ray brightness that contaminates the local background. For this reason, we used the high signal-to-noise background event files E1_fm0000_M1.fits, E1_00fm00_M2.fits, E1_0000fm_PN.fits (obtained using the medium filter) described in detail in Read \& Ponman (2003). The exposure and vignetting corrections were performed by dividing the superposed count images by the corresponding superposed exposure maps (produced using the task EEXPMAP). The pn exposure maps were scaled by the ratio of the pn/MOS effective areas to make MOS-equivalent superposed count rate images. We smoothed the images adaptively to a signal-to-noise ratio of 10 using the task ASMOOTH. The background-subtracted count images and the background- and continuum-subtracted line images (Sect. 3.2) were smoothed while accounting for their variances. The resulting template was then applied to smooth the associated exposure maps before performing the image division. The continuum under the lines was estimated by scaling a continuum band adjacent to the line emission using the continuum spectral slope derived from the global remnant spectrum. The energy ranges chosen for the lines and the continua are listed in Table 2. In all the line bands, the background is $\$ 0.5 \%$ of the total signal, except for the $7.65-8.0 \mathrm{keV}$ band where it is $\sim 3.6 \%$ of the total signal. In the continuum band 4.4-6.2 keV, the background amounts to $\sim 1.3 \%$. In all the images North is up and East is to the left.

For spectral analysis, we processed the event files with the EVIGWEIGHT task (Arnaud et al. 2001) to correct for vignetting effects. For the background subtraction, we used the same background event files used for the images. Spectra were rebinned to achieve a signal-to-noise ratio per bin $>5 \sigma$, and the spectral fits were performed simultaneously on the MOS1, MOS2, and pn spectra in the 1-9 keV band using XSPEC (Arnaud 1996). The chosen on-axis response files are m1,2_medv9q20t5r6_all_15.rsp for MOS1,2 spectra and epn_ff20_sdY9_medium.rsp for pn spectra. All the reported errors are at $90 \%$ confidence, as calculated according to Lampton et al. (1976).

\section{X-ray emission}

The broadband (1-9 keV) X-ray morphology of W49B obtained with the EPIC cameras is shown in Fig. 1 (left panel). For comparison, the VLA radio image obtained at $327 \mathrm{MHz}$ by Lacey et al. (2001) is also presented in Fig. 1 (right panel). The X-ray surface brightness of $\mathrm{W} 49 \mathrm{~B}$ has its maximum at the center of the remnant, and shows an elongated barrel-shaped structure extending for $\sim 5 \mathrm{pc}$ along its east-west axis (labelled $b$, see Fig. 1).

At the eastern end of the remnant, there is a bright elongated $\mathrm{X}$-ray structure (labelled $a$, see Fig. 1), running perpendicular to the X-ray barrel and parallel to the radio shell (labelled $\alpha$, see Fig. 1). The eastern side of $\mathrm{W} 49 \mathrm{~B}$ is bounded by a large molecular cloud detected in the $2.12 \mu \mathrm{m}$ molecular-hydrogen narrow-band image of W49B (Fig. 2). The X-ray barrel $b$ lies between two radio structures $(\beta$ and $\gamma$ ) that are parallel to the radio shell $(\alpha)$ and to the $\mathrm{H}_{2}$ emission. The positions of these structures $(\beta$ and $\gamma)$ are almost coincident with two coaxial ring-like structures observed in the $1.64 \mu \mathrm{m}$ [Fe II] image of W49B (Fig. 2). The X-ray structures $a$ (east) and $c$ (west) lie outside these radio structures. No intense X-ray emission has been detected in the south western region of the shell, where copious radio and $[\mathrm{Fe}$ II $]$ emission are observed ( $\delta$, see Fig. 1$)$. According to Lacey et al. (2001), there is evidence of stronger interstellar absorption in this direction. It is reasonable to conclude, however, that no hard X-ray emission is present in this region.

\subsection{Global spectrum}

The impressive global EPIC spectrum of W49B in the $1-9 \mathrm{keV}$ energy band is shown in Fig. 3. The spectrum is dominated by intense emission lines of He-like and $\mathrm{H}$-like ions of $\mathrm{Si}, \mathrm{S}, \mathrm{Ar}$ 

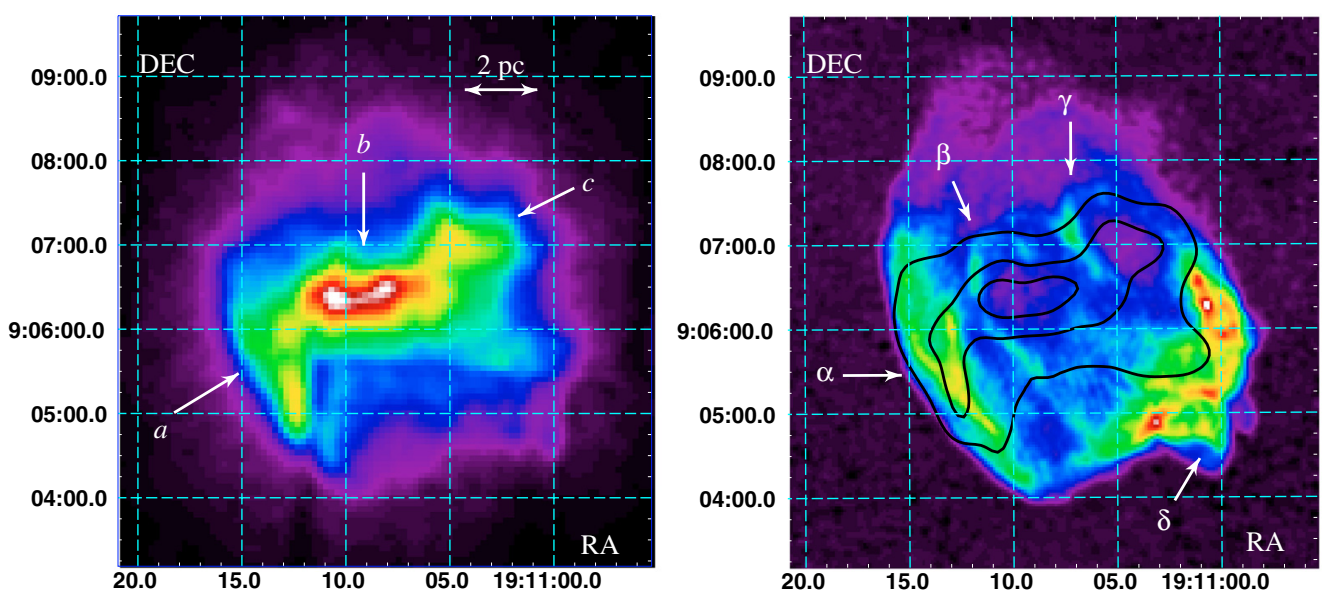

Fig. 1. Left panel: vignetting-corrected EPIC count-rate image of W49B in the $1-9 \mathrm{keV}$ band. The image is adaptively smoothed (with signal-tonoise ratio 10) and background-subtracted. The $2 \mathrm{pc}$ scale has been obtained assuming a distance of $8 \mathrm{kpc}$. The count-rate ranges between 0 and $7.2 \times 10^{-3} \mathrm{~s}^{-1}$. Right panel: radio image of W49B at $327 \mathrm{MHz}$ (angular resolution 6") obtained by Lacey et al. (2001). We have superimposed in black the X-ray contour levels derived from the 1-9 keV EPIC image at 25\%, 50\%, and 75\% of the maximum. The color bar has a linear scale.

Table 2. Parameters used for the production of the line images of Fig. 4 and the equivalent width maps of Fig. 5.

\begin{tabular}{lcc}
\hline \hline Emission line & Line energy band $(\mathrm{keV})$ & Continuum energy band $(\mathrm{keV})$ \\
\hline $\mathrm{S} \mathrm{XV} \mathrm{K} \alpha$ & $2.35-2.53$ & $2.75-3.0$ \\
$\mathrm{~S} \mathrm{XVI} \mathrm{K} \alpha$ & $2.58-2.7$ & $2.75-3.0$ \\
$\mathrm{Ar} \mathrm{XVII} \mathrm{K} \alpha$ & $3.05-3.2$ & $2.75-3.0$ \\
$\mathrm{Ca}$ XIX K $\alpha$ & $3.8-4.0$ & $3.45-3.7$ \\
$\mathrm{Fe} \mathrm{XXV} \mathrm{K} \alpha$ & $6.45-6.9$ & $4.4-6.2$ \\
$\mathrm{Fe} \mathrm{XXV+Ni} \mathrm{XXVII} \mathrm{K}$ & $7.65-8.0$ & $7.05-7.5$ \\
\hline
\end{tabular}

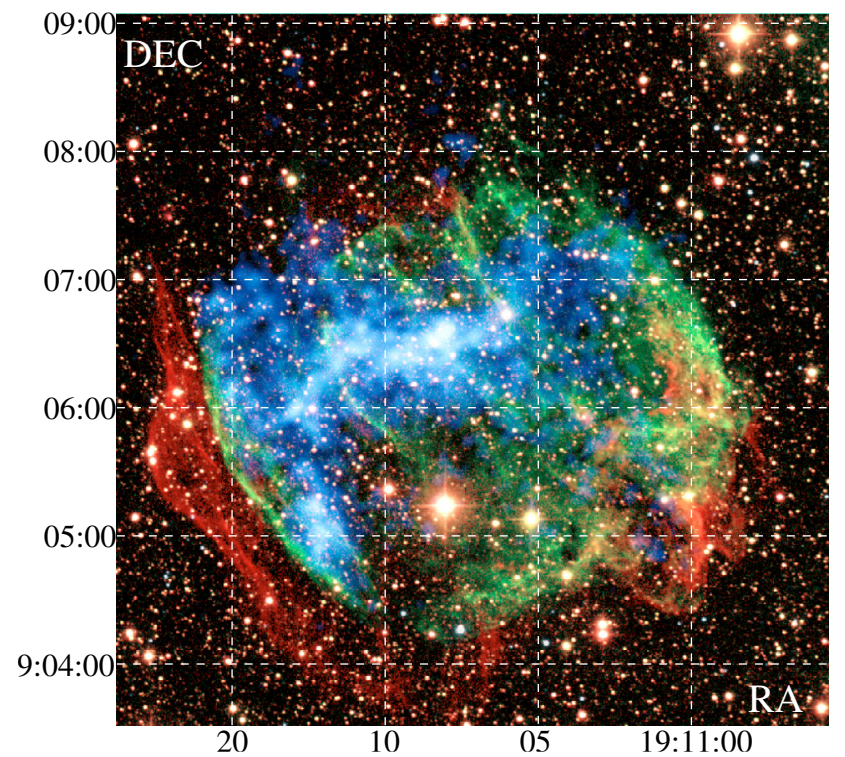

Fig. 2. Color composite Chandra X-ray image (in blue), infrared $2.12 \mu \mathrm{m}$ molecular hydrogen (in red), and $1.64 \mu \mathrm{m}$ [Fe II] (in green) images of W49B (from http://chandra.harvard.edu/ press/04_releases/press_060204.html).

and $\mathrm{Ca}$ and by a huge $\mathrm{Fe} \mathrm{K}$ emission line blend. A phenomenological modeling of the global spectrum ( 2 bremsstrahlung for the continuum + narrow Gaussians for the lines) allows us to derive the line energies reported in Fig. 3. The emission feature observed at $\sim 7.8 \mathrm{keV}$ corresponds to a possible blending of Fe XXV and Ni XXVII $\mathrm{K}$ emission lines and is discussed

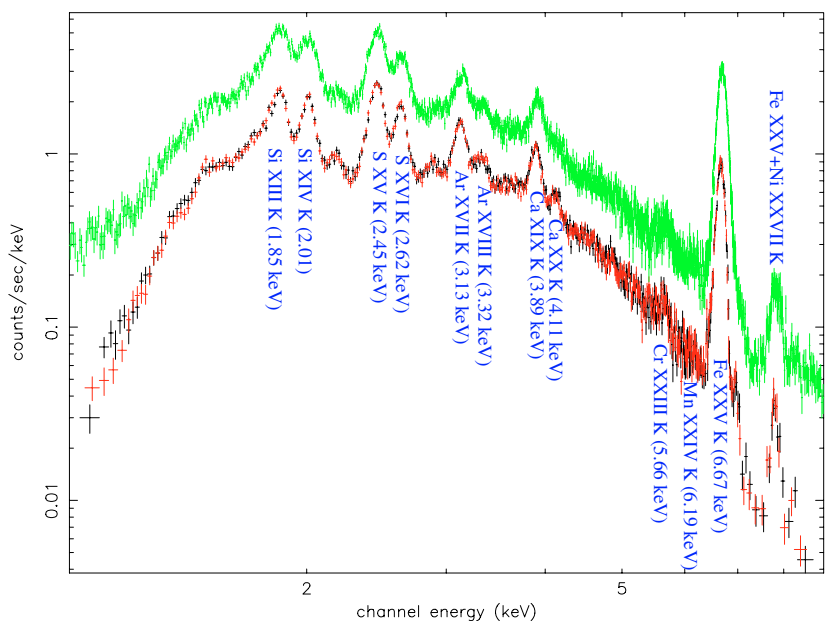

Fig. 3. pn (upper) and MOS (lower) global spectra of W49B in the 1-9 keV energy band, extracted from a circular region covering the entire remnant (of radius $3.3^{\prime}$ corresponding to $\sim 7.7 \mathrm{pc}$ ). Line energies have been determined using narrow Gaussian components on top of a thermal bremsstrahlung continuum. Note that to reproduce the continuum at energies below the $\mathrm{Si} \mathrm{K}$ lines, an additional bremsstrahlung component is required.

in Sect. 3.4. The continuum above $2.3 \mathrm{keV}$ is described well by a thermal bremsstrahlung model with $k T=1.69 \mathrm{keV}$ and $N_{\mathrm{H}}=5.3 \times 10^{22} \mathrm{~cm}^{-2}$; this model has been used to estimate the continuum under the lines given in Table 2 . Unlike the $A S C A$ spectrum (Hwang et al. 2000), the XMM-Newton global spectrum cannot be correctly fitted by a two-component thermal model (reduced $\chi^{2} \sim 2.4$ ). This indicates that the emission 
originates from physically inhomogeneous regions. Spatially resolved spectral analysis of homogeneous regions is required to correctly reproduce the spectra with 2-component models, as discussed in Sect. 3.3.

In the analysis of the ASCA global spectrum of W49B, Hwang et al. (2000) have found evidence of the presence of two new emission line features (at $\sim 5.7 \mathrm{keV}$ and $\sim 6.2 \mathrm{keV}$ ) that are not included in the currently available atomic database for X-ray spectral modeling. These were attributed to the He-like $\mathrm{Cr}$ transitions (forbidden line at $5.655 \mathrm{keV}$; resonance line at $5.682 \mathrm{keV}$ ) and the He-like Mn transitions (forbidden line at $6.151 \mathrm{keV}$; resonance line at $6.181 \mathrm{keV}$ ). Our XMM-Newton data confirm this detection. Modeling the global spectrum of the remnant in the 4.4-6.4 keV band with a thermal bremsstrahlung continuum, we found a clear excess in the residuals corresponding to these transitions. These lines can be reproduced by adding two Gaussian components to the bremsstrahlung model to determine the line energy and flux. We found, respectively for $\mathrm{Cr}$ and $\mathrm{Mn}$, line centroid energy $E_{\mathrm{Cr}}=5.66 \pm 0.01 \mathrm{keV}$ and flux $F_{\mathrm{Cr}}=2.5 \pm 0.4 \times 10^{-5}$ photons cm $\mathrm{cm}^{-2} \mathrm{~s}^{-1}, E_{\mathrm{Mn}}=6.19_{-0.04}^{+0.01} \mathrm{keV}$ and $F_{\mathrm{Mn}}=1.0 \pm 0.3 \times 10^{-5}$ photons $\mathrm{cm}^{-2} \mathrm{~s}^{-1}$, in good agreement with the values reported by Hwang et al. (2000): $E_{\mathrm{Cr}}=$ $5.69_{-0.03}^{+0.02} \mathrm{keV}$ and $F_{\mathrm{Cr}}=3.0_{-1.1}^{+0.8} \times 10^{-5}$ photons $\mathrm{cm}^{-2} \mathrm{~s}^{-1}$; $E_{\mathrm{Mn}}=6.17 \pm 0.05 \mathrm{keV}$ and $F_{\mathrm{Mn}}=1.3_{-0.6}^{+1.4} \times 10^{-5}$ photons $\mathrm{cm}^{-2} \mathrm{~s}^{-1}$. The addition of the $\mathrm{Cr}$ Gaussian leads to a reduction of $\chi^{2}, \delta \chi^{2}=111$ for 616 d.o.f.; adding the Mn Gaussian gives an additional $\delta^{\prime} \chi^{2}=28$ (to be compared with the values obtained with the $A S C A$ spectrum: $\delta \chi^{2}=20$ for 61 d.o.f. and $\left.\delta^{\prime} \chi^{2}=10\right)$. We found no indication of the presence of the Ti He $\alpha$ line (at $4.97 \mathrm{keV}$ ), obtaining an upper limit for the flux $F_{\mathrm{Ti}}<3.6 \times 10^{-6}$ photons $\mathrm{cm}^{-2} \mathrm{~s}^{-1}$, which is more stringent than the one reported from ASCA $\left(F_{\mathrm{Ti}}<2 \times 10^{-5}\right.$ photons $\mathrm{cm}^{-2} \mathrm{~s}^{-1}$, Hwang et al. 2000). The flux of the $\mathrm{Cr}$ and $\mathrm{Mn}$ lines contributes $\sim 3 \%$ of the total flux in the 4.4-6.4 band.

\subsection{Imaging}

The spatially resolved spectral study of the emission in the different lines provides information about the spatial distribution of the synthesized elements and the chemical composition of the $\mathrm{X}$-ray emitting plasma. On the basis of low angular resolution $A S C A$ images of W49B, Fujimoto et al. (1995) argued that the sulfur emission, unlike the iron emission, was associated with the outer envelope of the remnant and not related to the bright 4-6 keV continuum emission from the center.

To investigate both this claim and the suggested radial stratification of the elements, we produced background- and continuum-subtracted line images of $\mathrm{S}, \mathrm{Ar}, \mathrm{Ca}, \mathrm{Fe}$, and $\mathrm{Ni}$ lines (see Table 2 and Fig. 4). The Si emission line images shown in Fig. 6 are discussed in Sect. 3.3.1. The S H-like line image is very similar to the $\mathrm{S} \mathrm{He}$-like line image and therefore is not shown in the figure. All the line images present a bright centrally barrel-shaped morphology similar to the 4.4-6.2 keV continuum emission, as shown in Fig. 4. Overall, there are no significant differences between the morphologies in the $\mathrm{Si}, \mathrm{S}, \mathrm{Ar}, \mathrm{Ca}$, and $\mathrm{Fe}$ lines (although some detailed structures may differ as we discuss later). This result does not agree with the line-imaging results obtained for the lower-Z elements with ASCA data (see Fig. 4 in Fujimoto et al. 1995).

A detailed analysis of the Fe $\mathrm{K}$ line image in Fig. 4 (lower left panel) shows that the iron emission differs from that of lower- $Z$ elements in the western part of the remnant. In particular, regions 6 and 7 do not have significant Fe line emission, while they do have significant emission from $\mathrm{Si}, \mathrm{S}, \mathrm{Ar}$, and $\mathrm{Ca}$. The lower statistic Fe XXV+Ni XXVII image of Fig. 4 (lower right panel) presents a morphology that is fully consistent with the Fe K image.

To disentangle higher element abundances in the bright line image structures from higher emission measure (which might be caused by either higher density or higher emission volume), we produced equivalent width maps as shown in Fig. 5. These depend linearly on the abundances, but are also affected by the temperature, column density, and ionization age. These images were constructed by dividing the background- and continuum-subtracted line images by the corresponding underlying continuum. Before the division, the estimated continuum map was adaptively smoothed using the same template as used for the background- and continuum-subtracted line images. As explained in Sect. 2, the underlying continuum is estimated from continuum regions adjacent to the line emission, under the assumption that the continuum has a uniform spectral slope over the entire SNR. As shown in Fig. 5, the S map is clearly different from the other maps, but the equivalent width images of $\mathrm{Ar}, \mathrm{Ca}$, $\mathrm{Fe}$, and $\mathrm{Fe}+\mathrm{Ni}$ all show similar features. In particular, all these maps show higher equivalent widths in the central and eastern parts of the remnant than in the western part, with maxima corresponding approximately to regions 1 and 3 . This is particularly true for iron. In the Ar map there is an indication of nonnegligible equivalent widths in the western regions 6 and 7.

As the S H-like and He-like line images are very similar, we used the combined $2.35-2.7 \mathrm{keV}$ band to improve the statistics of the sulfur equivalent width map as shown in Fig. 5 (upper left panel). The map appears patchy and shows no clear structures. Given the relatively low line energies, the map may be affected by local variations of $N_{\mathrm{H}}$, as we shall see in Sect. 3.3.1.

In conclusion, the line emission maps of all the observed elements show a centrally elongated bright structure in good agreement with the morphology of the continuum. The equivalent width images indicate that $\mathrm{Ar}, \mathrm{Ca}$, and $\mathrm{Fe}$ may be more abundant in the central and eastern parts of the remnant than in the west. The high values of the equivalent width are therefore not restricted to the barrel-like structure, but extend to the eastern side of the remnant, as illustrated in Fig. 5 (lower right panel), where we show the contours of the iron line image superimposed on the iron equivalent width map. A clear drop-off of the equivalent width is observed towards the western side. This effect is particularly strong for iron, and is less pronounced for the lower- $Z$ elements, which show significant equivalent widths in the west. While we thus find an East/West abundance anisotropy, we find no clear evidence of radial stratification of the elements.

\subsection{Spatially resolved spectral analysis}

To quantify the equivalent width map results for the element distributions, we performed a spatially resolved spectral analysis that allowed us to study the physical and chemical characteristics of the plasma in detail in different parts of the remnant. In particular, we analyzed the spectra extracted from seven fairly homogeneous regions shown in Fig. 4 (lower left panel) and in Fig. 5. While this set of regions does not cover the entire remnant, it does allow us to study the spatial variations of the spectral properties in the X-ray emitting plasma. In order to analyze spectra extracted from physically uniform regions, we produced a MOS mean photon energy map (i.e., an image where each pixel holds the mean energy of the detected MOS photons) in the continuum band 4.4-6.2 keV. The shape and size of the seven regions 

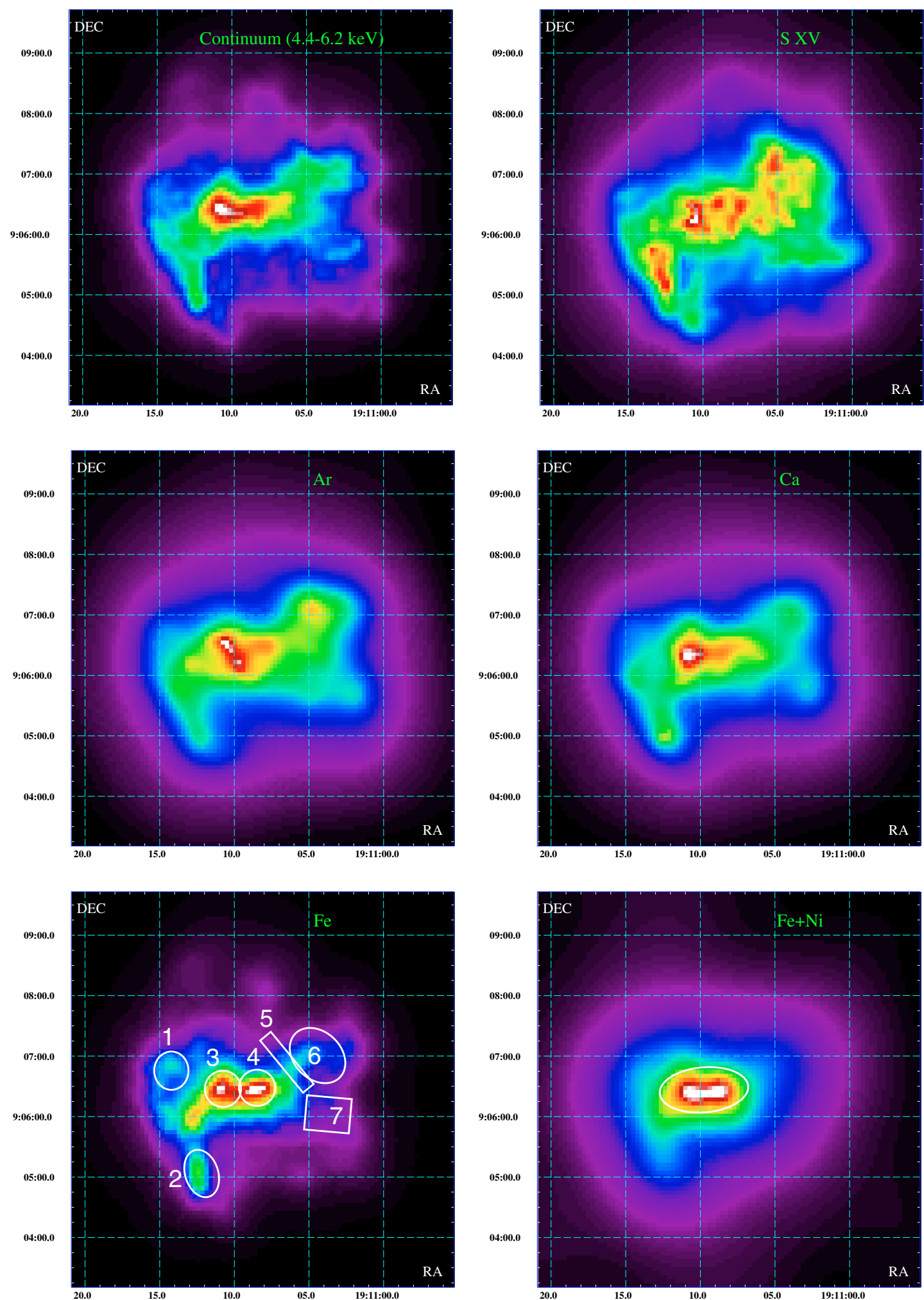

Fig. 4. Vignetting-corrected and continuum-subtracted EPIC count rate images of the continuum (4.4-6.2 keV, upper left), S XV K (2.35-2.53 keV, upper right), Ar XVII K (3.05-3.2 keV, central left), Ca XIX K (3.8-4.0 keV, central right), Fe XXV K (6.45-6.9 keV, lower left), and Fe XXV+Ni XXVII K (7.65-8.0 keV, lower right). All images are adaptively smoothed with signal-to-noise ratio 10 . The 7 regions selected for spatially resolved spectral analysis are shown in the Fe XXV K line image, while the ellipse in the Fe XXV+Ni XXVII K line image indicates the region used for the detection of the Ni line (Sect. 3.4). The count rates range between 0 and: $8.6 \times 10^{-4} \mathrm{~s}^{-1}(\mathrm{continuum}) ; 3.4 \times 10^{-4} \mathrm{~s}^{-1}(\mathrm{~S})$; $1.7 \times 10^{-4} \mathrm{~s}^{-1}(\mathrm{Ar}) ; 1.9 \times 10^{-4} \mathrm{~s}^{-1}(\mathrm{Ca}) ; 7.0 \times 10^{-4} \mathrm{~s}^{-1}(\mathrm{Fe}) ; 2.1 \times 10^{-5} \mathrm{~s}^{-1}(\mathrm{Fe}+\mathrm{Ni})$. The color bar has a linear scale. 

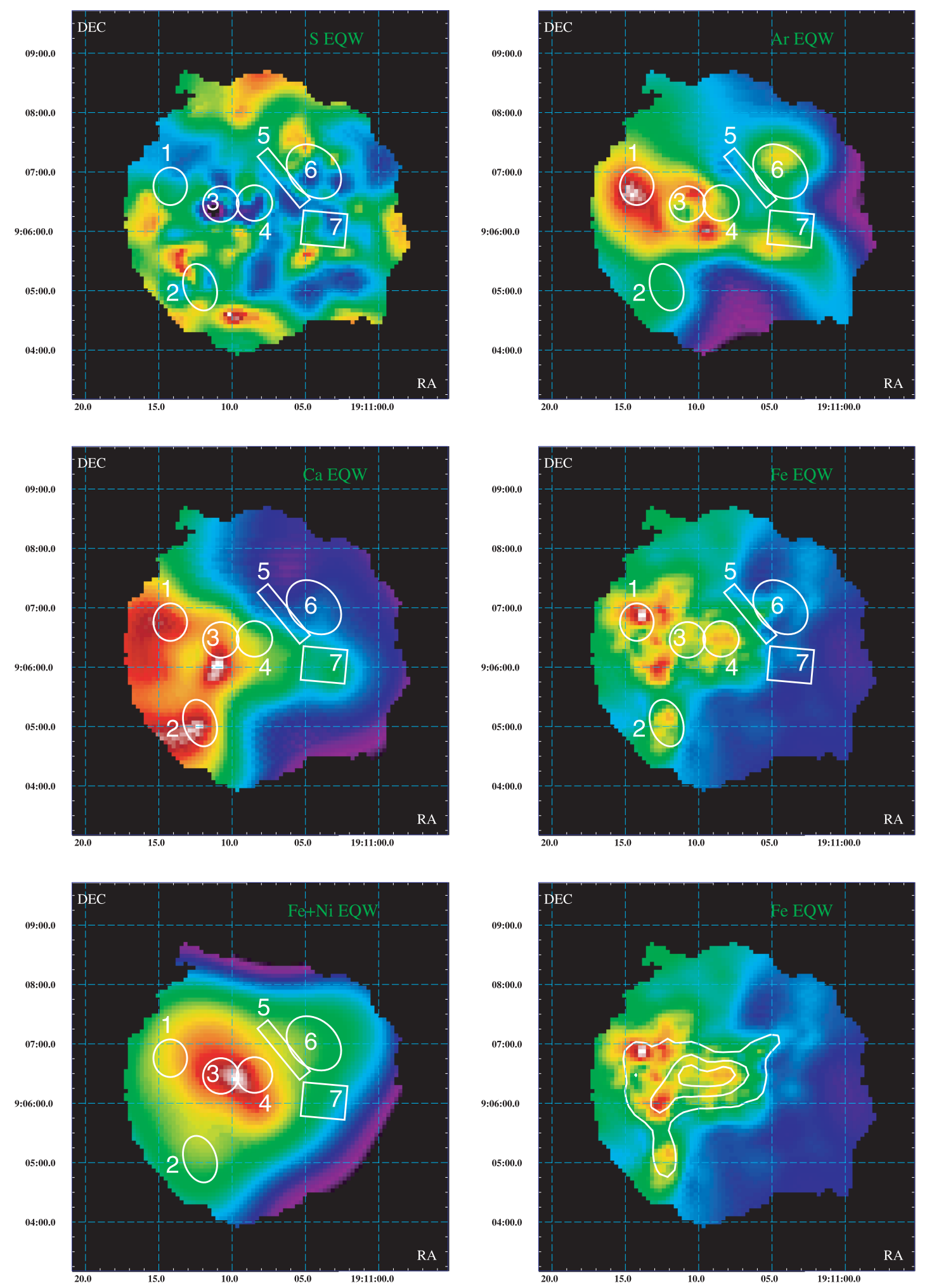

Fig. 5. Equivalent-width maps of the S He-like and H-like line emission in the 2.35-2.7 keV band (upper left), of the Ar XVII K blend (3.05-3.2 keV, upper right), of Ca XIX K (2.35-2.7 keV, central left), of Fe XXV K (6.45-6.9 keV, central right), and of the Fe XXV+Ni XXVII K emission lines in the 7.65-8.0 keV band (lower left). In the lower right panel we have overlaid the $75 \%$, 50\%, and $25 \%$ contour levels of the corresponding line image (Fig. 4, lower left panel) on the Fe XXV K equivalent width map. The ranges of the equivalent width maps are: $0.245-0.6 \mathrm{keV}(\mathrm{S}), 0.07-0.13 \mathrm{keV}(\mathrm{Ar}), 0.085-0.18 \mathrm{keV}(\mathrm{Ca}), 0-12.1 \mathrm{keV}(\mathrm{Fe}), 0.315-1.4 \mathrm{keV}(\mathrm{Fe}+\mathrm{Ni})$. 


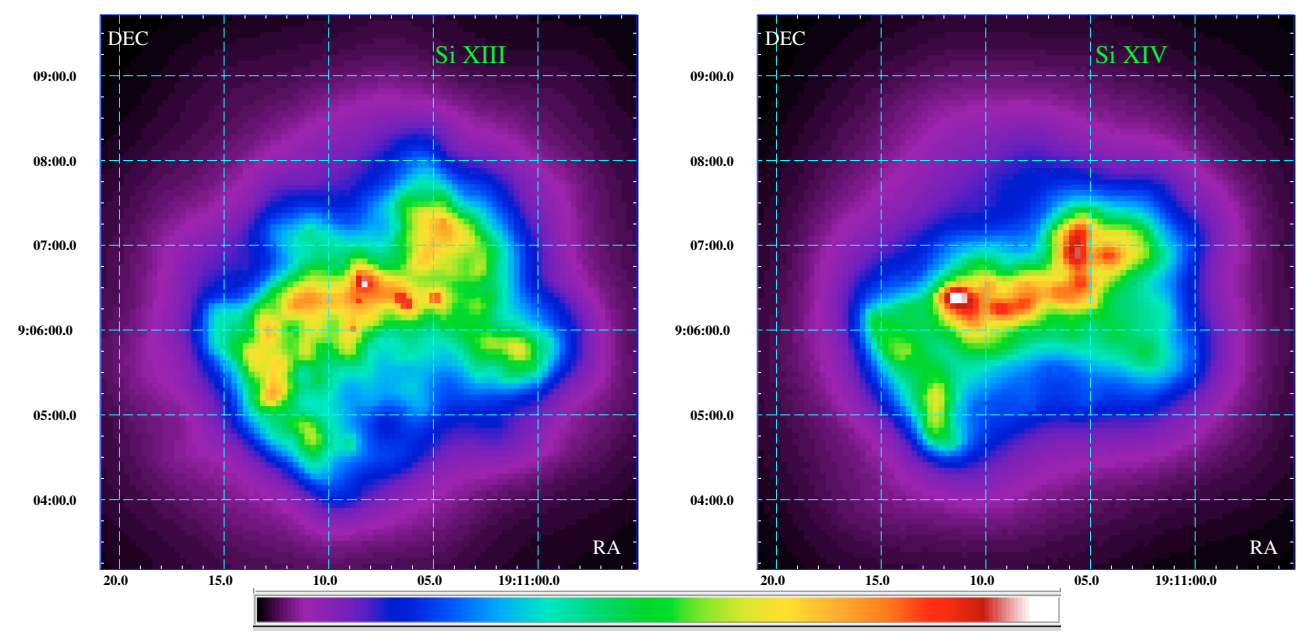

Fig. 6. Vignetting-corrected and continuum-subtracted EPIC count rate image of the Si XIII K $\alpha$ (1.77-1.9 keV, left panel) and Si XIV K $\alpha$ (1.96-2.06 keV, right panel). The images are adaptively smoothed with signal-to-noise ratio 10 . The count rates range between 0 and $2.3 \times 10^{-4} \mathrm{~s}^{-1}$ for Si XIII and $1.7 \times 10^{-4} \mathrm{~s}^{-1}$ for Si XIV. The color bar has a linear scale.
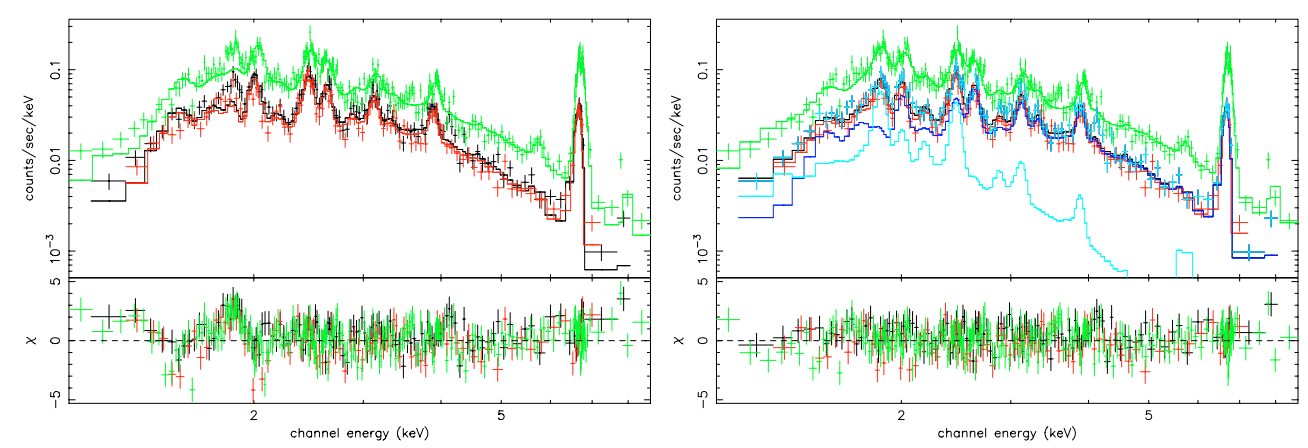

Fig. 7. pn (upper) and MOS (lower) spectra of region 2 with the corresponding best-fit model and residuals obtained with one thermal component (left panel) and with two thermal components (right panel). The contribution of the two components for the MOS camera is indicated in the right panel in cyan for the cooler component and blue for the hotter component.

were chosen in order to have very small fluctuations of the mean photon energy $(\sim 1 \%)$ and a sufficient number of photons.

Regions 1 and 2 are located on the X-ray eastern, elongated structure along the radio shell (labelled $a / \alpha$ in Fig. 1), at its northern and southern ends, respectively. They both exhibit high values in the equivalent width maps. Regions 3 and 4 cover the brightest part of the centrally barrel-shaped X-ray structure: region 3 corresponds to the maximum of the continuum emission in the 4.4-6.2 keV band (Fig. 4, upper left panel), and region 4 corresponds to the highest $\mathrm{Fe} \mathrm{K}$ line emission region (Fig. 4, lower left panel). Region 5 follows the western radio structure $\gamma$ (Fig. 1). Region 6 covers the western X-ray structure $c$ and, finally, region 7 lies on the faint south western structure that is seen in the continuum and $\mathrm{K}$ line emission of $\mathrm{S}, \mathrm{Ar}, \mathrm{Ca}$, but not of iron.

\subsubsection{Spectral modeling and results}

We first modeled the extracted spectra using XSPEC with a single MEKAL model of an optically-thin thermal plasma in collisional ionization equilibrium (Mewe et al. 1985, 1986; Liedahl et al. 1995). Good fits to the spectra require non-solar abundances for $\mathrm{Si}, \mathrm{S}, \mathrm{Ar}, \mathrm{Ca}$, and Fe. All other element abundances were fixed at their solar values. To account for the contribution from the $\mathrm{Cr}$ line (Sect. 3.1), we added a narrow Gaussian component with fixed energy; it was not necessary to account for the
Mn line. We also added a systematic 5\% error term to reflect the estimated $5-10 \%$ uncertainties in the calibration of the instrumental effective area. The reduced $\chi^{2}$ values obtained using the two MOS and the pn cameras range from 1.3 (with 354 d.o.f.), for region 5, to 1.7 (445 d.o.f.) for region 2. Above $2 \mathrm{keV}$, the models describe the continuum and emission line spectra quite well, but these single-temperature models severely underpredict the Si XIII K $\alpha$ line, as illustrated in Fig. 7 (left panel). The bestfit parameters for these fits are thus not reported here. The same problem was encountered by Hwang et al. (2000) in the modeling of the $A S C A$ global spectrum. We found that the quality of the fits does not significantly improve if a single non-equilibrium ionization (NEI) model is used instead.

We obtained a good description of the spectra by adding a second thermal MEKAL component in collisional ionization equilibrium. We required the two components to have the same abundances and to be absorbed by the same interstellar column density. The spectra are also well-described if the soft component has solar abundances and is in nonequilibrium ionization (PSHOCK model). In this case the temperatures of the two components (NEI+CIE) are comparable to those obtained for the two CIE components, but the fits give somewhat higher chisquared values. For example, in region $2: \chi_{\mathrm{NEI}+\mathrm{CIE}}^{2}=529.7$ (with 442 d.o.f.) and $\chi_{2 \mathrm{CIE}}^{2}=506.7$ (443); in region 4: $\chi_{\mathrm{NEI}+\mathrm{CIE}}^{2}=$ $821.2(673)$ and $\chi_{2 \mathrm{CIE}}^{2}=793.5(674)$; in region $6: \chi_{\mathrm{NEI}+\mathrm{CIE}}^{2}=$ $1064.1(843)$ and $\chi_{2 \mathrm{CIE}}^{2}=1059.3(844)$. We therefore adopt 
Table 3. Best-fit parameters for the spectra extracted in the seven regions shown in Fig. 4 (lower left panel), described with two thermal components in collisional ionization equilibrium. All errors are at the $90 \%$ confidence level.

\begin{tabular}{|c|c|c|c|c|c|c|c|}
\hline Parameter & Region 1 & $\overline{R e g i o n 2}$ & Region 3 & Region 4 & Region 5 & Region 6 & Region 7 \\
\hline$N_{\mathrm{H}}\left(10^{22} \mathrm{~cm}^{-2}\right)$ & $4.5 \pm 0.1$ & $4.22_{-0.05}^{+0.08}$ & $4.8_{-0.2}^{+0.1}$ & $4.5 \pm 0.1$ & $4.3_{-0.1}^{+0.2}$ & $4.61 \pm 0.05$ & $4.36_{-0.09}^{+0.08}$ \\
\hline$k T_{1}(\mathrm{keV})$ & $0.90_{-0.06}^{+0.08}$ & $0.75_{-0.05}^{+0.03}$ & $0.92_{-0.08}^{+0.2}$ & $0.8 \pm 0.1$ & $0.96_{-0.09}^{+0.1}$ & $0.78_{-0.04}^{+0.06}$ & $0.82_{-0.06}^{+0.09}$ \\
\hline$E M_{1}^{*}\left(10^{20} \mathrm{~cm}^{-5}\right)$ & $0.5_{-0.1}^{+0.06}$ & $1.16_{-0.09}^{+0.05}$ & $1.2 \pm 0.2$ & $1.3_{-0.3}^{+0.3}$ & $0.8_{-02}^{+0.09}$ & $1.1_{-02}^{+0.04}$ & $\begin{array}{l}0.92_{-0.05}^{+0.06} \\
0.16\end{array}$ \\
\hline$k T_{2}(\mathrm{keV})$ & $3.0_{-0.2}^{+0.4}$ & $2.19_{-0.06}^{+0.19}$ & $2.6 \pm 0.2$ & $2.35_{-0.09}^{+0.12}$ & $2.2_{-0.2}^{+0.2}$ & $1.77_{-0.09}^{+0.23}$ & $2.00 \pm 0.04$ \\
\hline$E M_{2}^{*}\left(10^{20} \mathrm{~cm}^{-5}\right)$ & $0.5 \pm 0.1$ & $1.1 \pm 0.1$ & $1.8_{-0.1}^{+0.1}$ & $1.95_{-0.08}^{+0.19}$ & $1.4 \pm 0.3$ & $2.2_{-0.1}^{+0.09}$ & $1.3 \pm 0.1$ \\
\hline $\mathrm{Si} / \mathrm{Si}_{\odot}$ & $2.2 \pm 0.4$ & $3.4_{-0.4}^{+0.2}$ & $3.7 \pm 0.4$ & $2.9 \pm 0.3$ & $2.4_{-0.5}^{+0.3}$ & $2.3 \pm 0.1$ & $2.1_{-0.2}^{+0.3}$ \\
\hline $\mathrm{S} / \mathrm{S}_{\odot}$ & $4.1 \pm 0.5$ & $4.1 \pm 0.3$ & $4.6_{-06}^{+0.3}$ & $3.6_{-0.4}^{+0.3}$ & $2.7_{-0.5}^{+0.5}$ & $2.4_{-0.1}^{+0.2}$ & $2.8 \pm 0.3$ \\
\hline $\mathrm{Ar} / \mathrm{Ar}_{\odot}$ & $5.6_{-0.9}^{+1.1}$ & $3.8 \pm 0.6$ & $4.9_{-0.6}^{+0.8}$ & $4.1_{-0.7}^{+0.4}$ & $2.6 \pm 0.6$ & $2.8 \pm 0.3$ & $3.4_{-0.5}^{+0.3}$ \\
\hline $\mathrm{Ca} / \mathrm{Ca}_{\odot}$ & $8 \pm 1$ & $6.6_{-0.8}^{+0.9}$ & $7 \pm 1$ & $6.9_{-0.9}^{+0.7}$ & $4.7_{-0.9}^{+0.8}$ & $4.4_{-0.4}^{+0.4}$ & $5.2^{-0.5}$ \\
\hline $\mathrm{Fe} / \mathrm{Fe}_{\odot}$ & $5.2_{-0.4}^{+0.6}$ & $5.1 \pm 0.3$ & $5.1_{-0.6}^{+0.4}$ & $5.6_{-0.3}^{+0.4}$ & $2.6_{-0.3}^{+0.9}$ & $2.5_{-0.2}^{-0.5}$ & $1.6_{-0.1}^{+0.7}$ \\
\hline$\chi^{2} /$ d.o.f. & $298.8 / 244$ & $506.7 / 443$ & $800.0 / 646$ & $793.5 / 674$ & $403.2 / 352$ & $1059.3 / 844$ & $566.3 / 434$ \\
\hline$f_{1}$ & $0.08_{-0.04}^{+0.03}$ & $0.11_{-0.03}^{+0.04}$ & $0.08 \pm 0.04$ & $0.08 \pm 0.03$ & $0.10_{-0.05}^{+0.06}$ & $0.09_{-0.04}^{+0.06}$ & $0.11_{-0.03}^{+0.04}$ \\
\hline$n_{1}\left(\mathrm{~cm}^{-3}\right)$ & $6_{-2}^{+3.04}$ & $7_{-1}^{+2.03}$ & $7_{-2}^{+4}$ & $8 \pm 2$ & $5_{-2}^{+3.05}$ & $7_{-2}^{+4.04}$ & $5.5_{-0.9}^{+3.03}$ \\
\hline$n_{2}\left(\mathrm{~cm}^{-3}\right)$ & $1.8_{-0.2}^{+0.3}$ & $2.5_{-0.1}^{+0.2}$ & $2.6 \pm 0.2$ & $2.6_{-0.1}^{+0.3}$ & $2.3 \pm 0.2$ & $3.0_{-0.2}^{+0.8}$ & $2.3 \pm 0.2$ \\
\hline
\end{tabular}

* Emission measure per unit area.

the two CIE component model hereafter, while cautioning the reader that the high interstellar absorption complicates the description of the soft component and hence the discrimination between these two models.

Figure 7 shows a representative spectrum, extracted from region 2, with the best-fit model and residuals for two thermal components (right panel) and, for comparison, a single thermal component (left panel). The best-fit parameters for all seven spectral regions are given in Table 3 .

The values of $N_{\mathrm{H}}$ in the different spectral regions range approximately from $4.2 \times 10^{22} \mathrm{~cm}^{-2}$ to $4.9 \times 10^{22} \mathrm{~cm}^{-2}$ (compared with the global $N_{\mathrm{H}}=5.0_{-0.2}^{+0.3} \times 10^{22} \mathrm{~cm}^{-2}$ estimated by Hwang et al. 2000). To produce the line and the equivalent-width images (see Sect. 3.2), we estimated the continuum contribution under the lines by assuming a uniform absorbed slope of the continuum based on the global spectrum of W49B. For low energy lines, spatial variation of the column density over the remnant can thus introduce errors in the line and equivalent width images. In particular, where $N_{\mathrm{H}}$ is higher than its global averaged value, we may overestimate the continuum under the line and hence underestimate the equivalent width. We observe this kind of effect in the S equivalent width image (Fig. 5, upper left panel), where there is a minimum in region 3 with a mean value of $0.32 \mathrm{keV}$, corresponding to the highest value of the column $\left(N_{\mathrm{H}}=4.8_{-0.2}^{+0.1}\right)$, and a higher mean equivalent width of $0.4 \mathrm{keV}$ in region 2 , which has a lower column $\left(N_{\mathrm{H}}=4.22_{-0.05}^{+0.08}\right)$.

Variations in column density may also affect the Si images, but in this case there is no clear relation between such variations and inhomogeneities in the equivalent-width map. This probably indicates that other effects are also present. As explained above, a single temperature model provides a good overall description of the spectra in the $1-9 \mathrm{keV}$ band, but fails to reproduce the observed $\mathrm{Si}$ line flux in the narrow energy range 1.7-1.9 keV. It is therefore possible that the Si He-like emission originates predominantly in a plasma with different physical conditions than the one responsible for the other emission lines. As shown in Fig. 6, the He-like line morphology resembles that of the Si H-like emission, as well as the S, Ar, and Ca line emission. The Si equivalent width maps (not presented here) have irregular patchy features at odds both with those seen for the other elements and with the spatially resolved spectral analysis. This is probably because the continuum of the hard component is not negligible at low energies, even though the Si He-like line emission itself is associated with the soft thermal component (see the right panel of Fig. 7).

We observe larger variations for the temperature of the hotter component $k T_{2}(1.75-3.3 \mathrm{keV})$ than for the cooler component $k T_{1}(0.7-1.05 \mathrm{keV})$, but do not measure any radial temperature structure (see Table 3). The temperature of the hotter component is at a maximum in region 1 at $k T_{2}$, and decreases significantly moving southward along the X-ray elongated $a$ structure to region 2. This temperature also decreases towards the western side of the remnant (from region 3 to region 6). We observe that the difference between the continuum surface brightness of region 3 (i.e. the brightest region in the continuum band 4.4-6.2 $\mathrm{keV}$ ) and region 4 is caused by the higher temperature in region 3 , and not by differences in the emission measure.

In Table 3, the parameter EM indicates the values of the emission measure per unit area, which is the product of the square of the particle density times the extension of the plasma along the line of sight. These values are in the range $0.3-1.7 \times 10^{20} \mathrm{~cm}^{-5}$ for the cooler component, and $0.4-3.5 \times 10^{20} \mathrm{~cm}^{-5}$ for the hotter component. In region 6 we have the highest values for both $E M_{1}$ and $E M_{2}$. Associated with the decreasing trend of $T_{2}$, we note that the corresponding emission measure $E M_{2}$ is slightly higher in region 6 than in regions 3 and 4.

We used the best-fit values of temperature and emission measure to derive the plasma density (indicated with $n$ in Table 3) and the filling factor $(f)$ of each component, obtained as in Bocchino et al. (1999). These quantities were derived by assuming pressure equilibrium between the two components and assuming that in each spectral region the extension of the X-ray emitting plasma is equal to the length of the chord intercepted by the radio shell (approximated as a sphere with angular radius $\phi=2.15^{\prime}$ ) along the line of sight. The filling factor of the cool component is $\lesssim 0.1$, and the densities of the two components are almost uniform across the remnant.

We found significant element overabundances in all the spectral regions: all the elements have fitted abundances $>1.5$ (at $90 \%$ confidence level) with respect to their solar value. This attests to the origin of the emission in the ejecta. Moreover, higher abundance values are found for the central and eastern regions compared to the western regions, in good 
agreement with the results of our image analysis. All abundances are lower in regions 5, 6, and 7, with the largest decrease observed for the $\mathrm{Fe}$ abundance, as expected. For example, the ratio $\left(\mathrm{Ar} / \mathrm{Ar}_{\odot}\right) /\left(\mathrm{Fe} / \mathrm{Fe}_{\odot}\right)$ is $0.7 \pm 0.1$ in region 4 and $1.8 \pm 0.3$ in region 6 , while the ratio $\left(\mathrm{Ca} / \mathrm{Ca}_{\odot}\right) /\left(\mathrm{Fe} / \mathrm{Fe}_{\odot}\right)$ is $1.2 \pm 0.2$ in region 4 and $1.8_{-0.2}^{+0.3}$ in region 6 . This agrees with the considerations discussed at the end of Sect. 3.2, where regions 6 and 7 show low equivalent widths for iron, but nonnegligible line emission and line equivalent widths for the lower-Z elements. All the abundances reported in Table 3 are given as the ratio of the number density of the respective element to the number density of hydrogen expressed in units of the solar value of this ratio. In a pure-ejecta plasma (where hydrogen emission is negligible), this ratio would be ill-defined, and the spectral shape would only depend on the relative abundances of the heavy elements. We verified that this is not the case for our fits. In fact if we scale all the abundances by the same factor $F$, we obtain significantly higher $\chi^{2}$ values for $F \gtrsim 1.5$. Therefore the abundances in Table 3 are well-defined and above solar.

In each spectral region we also obtained another $\chi^{2}$ minimum with $k T_{1} \sim 0.2 \mathrm{keV}$. This value agrees with the results obtained with the ASCA data by Hwang et al. (2000) and Kawasaki et al. (2005). In this case, however, we found very high values for the emission measure per unit area of the cool component $\left(E M_{1} \gtrsim 10^{22} \mathrm{~cm}^{-5}\right)$, which would imply unrealistically high values for the filling factor $\left(f_{1} \sim 0.5\right)$ and the density $\left(n_{1} \sim 50 \mathrm{~cm}^{-3}\right)$. In what follows, we therefore only discuss the results presented in Table 3.

\subsection{Evidence of Ni overabundance}

Thanks to the high $X M M-N e w t o n$ effective area at high energies, it was possible to detect a strong emission feature at $\sim 7.8 \mathrm{keV}$, with possible contributions from both Fe XXV and Ni XXVII. The Ni XXVII emission line has been previously detected in only one SNR, Cas A (Bleeker et al. 2001; Willingale et al. 2002). This emission blend can be used to set constraints on the Ni abundance in W49B. Since the photon statistics for the seven individual spectra do not allow us to resolve the features in the $7.65-8.0 \mathrm{keV}$ band in detail, we extracted the spectrum from the larger elliptical region shown in Fig. 4 (lower right panel). This region covers the area of highest surface brightness in the 7.65-8.0 keV band. We fitted the spectrum in the 1-9 keV band with the 2-component thermal model used for the spatially resolved spectral analysis. Table 4 gives the best-fit parameters derived both by fixing the $\mathrm{Ni}$ abundance to the solar value (second column) and by fitting the $\mathrm{Ni}$ abundance as a free parameter (third column). We can reproduce the Fe XXV and Ni XXVII K line blends better by fitting for the Ni abundance. The F-test gives the probability that the improvement of the fit is insignificant as $<10^{-10}$. We conclude that there is convincing evidence of $\mathrm{Ni}$ overabundance $\left(\mathrm{Ni} / \mathrm{Ni}_{\odot}=10_{-1}^{+2}\right)$ in the centrally barrel-shaped structure of W49B.

\subsection{Overionization}

Through their analysis of the $A S C A$ global spectrum, Kawasaki et al. (2005) claimed the presence of "overionized" plasma in W49B. In particular, they measured the intensity ratio of the $\mathrm{H}$-like to the He-like $\mathrm{K} \alpha$ lines of $\mathrm{Ar}$ and $\mathrm{Ca}$ to obtain the ionization temperature $T_{z}$, and found that $T_{z}$ is higher than the electron temperature $T_{\mathrm{e}}$ estimated from the continuum of the $\mathrm{X}$-ray spectrum. We performed the same analysis by modeling
Table 4. Best-fit parameters for the spectrum extracted from the elliptical region shown in Fig. 4 (lower right panel) and reproduced by two thermal components in collisional ionization equilibrium. All errors are at the $90 \%$ confidence level.

\begin{tabular}{lcc}
\hline \hline Parameter & $\mathrm{Ni} / \mathrm{Ni}_{\odot}=1$ & $N i$ thawed \\
\hline$N_{\mathrm{H}}\left(10^{22} \mathrm{~cm}^{-2}\right)$ & $4.60_{-0.03}^{+0.06}$ & $4.68_{-0.02}^{+0.06}$ \\
$k T_{1}(\mathrm{keV})$ & $0.87_{-0.02}^{+0.04}$ & $0.86_{-0.05}^{+0.02}$ \\
$E M_{1}^{*}\left(10^{20} \mathrm{~cm}^{-5}\right)$ & $1.13_{-0.02}^{+0.04}$ & $1.1_{-0.2}^{+0.1}$ \\
$k T_{2}(\mathrm{keV})$ & $2.35_{-0.03}^{+0.02}$ & $2.2_{-0.1}^{+0.2}$ \\
$E M_{2}^{*}\left(10^{20} \mathrm{~cm}^{-5}\right)$ & $1.79_{-0.04}^{+0.06}$ & $1.82_{-0.05}^{+0.06}$ \\
$\mathrm{Si} / \mathrm{Si}_{\odot}$ & $3.3_{-0.3}^{+0.1}$ & $3.3 \pm 0.2$ \\
$\mathrm{~S} / \mathrm{S}_{\odot}$ & $3.7 \pm 0.1$ & $3.7_{-0.2}^{+0.1}$ \\
$\mathrm{Ar} / \mathrm{Ar}_{\odot}$ & $4.2_{-0.2}^{+0.4}$ & $4.2_{-0.4}^{+0.3}$ \\
$\mathrm{Ca} / \mathrm{Ca}_{\odot}$ & $6.3 \pm 0.5$ & $6.4 \pm 0.5$ \\
$\mathrm{Fe} / \mathrm{Fe}_{\odot}$ & $5.5_{-0.2}^{+0.1}$ & $6.0_{-0.2}^{+0.1}$ \\
$\mathrm{Ni} / \mathrm{Ni}_{\odot}$ & 1 & $10_{-1}^{+4}$ \\
$\chi^{2} /$ d.o.f. & $1847.8 / 1327$ & $1584.3 / 1326$ \\
\hline
\end{tabular}

* Emission measure per unit area.

the XMM-Newton global spectrum in the $2.75-6.0 \mathrm{keV}$ band. We modeled the continuum and estimate $T_{\mathrm{e}}$ with a zero metallicity VMEKAL component and included four narrow Gaussian components to model the Ar and Ca lines and a fifth Gaussian component for the $\mathrm{Cr}$ line. We considered absorption column densities of $N_{\mathrm{H}}=4.6 \times 10^{22} \mathrm{~cm}^{-2}$ and $N_{\mathrm{H}}=5.23 \times 10^{22} \mathrm{~cm}^{-2}$ (the value adopted by Kawasaki et al. 2005). For those column densities, we obtained electron temperatures of $k T_{\mathrm{e}}=1.80 \pm 0.03 \mathrm{keV}$ and $1.66_{-0.01}^{+0.02} \mathrm{keV}$, respectively. We find no evidence of overionization for Ar, given the respective ionization temperatures of $k T_{z}=1.68 \pm 0.07$ and $k T_{z}=1.64_{-0.05}^{+0.08}$. For Ca, the ionization temperature does seem higher than the continuum temperature: $k T_{z}=2.0_{-0.05}^{+0.1}$ with $N_{\mathrm{H}}=4.6 \times 10^{22} \mathrm{~cm}^{-2}$ and $k T_{z}=2.04_{-0.08}^{+0.1}$ with $N_{\mathrm{H}}=5.23 \times 10^{22} \mathrm{~cm}^{-2}$. However, we caution the reader that these estimates may not be reliable because the global spectrum originates from physically non-uniform regions of W49B that have different temperatures and abundances. A case in point is that the estimates of the electron temperatures obtained from the global spectrum are significantly lower than those obtained from our spatially resolved spectral analysis of homogeneous regions (see Sect. 3.3 and $k T_{2}$ values in Table 3 ). We therefore repeated our analysis for the more uniform central region indicated by a white ellipse in the lower left panel of Fig. 5. This is where the overionization effects are expected to be strongest in the scenario proposed by Kawasaki et al. (2005). Our analysis shows that these effects are not present. Using the same model described above in the 2.75-6.0 keV band, we found for $N_{\mathrm{H}}=$ $4.6 \times 10^{22} \mathrm{~cm}^{-2}$ and $N_{\mathrm{H}}=5.23 \times 10^{22} \mathrm{~cm}^{-2}$ : electron temperatures of $k T_{\mathrm{e}}=2.4 \pm 0.1 \mathrm{keV}$ and $k T_{\mathrm{e}}=2.25_{-0.1}^{+0.08}$, Ar ionization temperatures of $k T_{z}=1.9_{-0.2}^{+0.1}$ and $k T_{z}=1.8 \pm 0.1$, and Ca ionization temperatures of $k T_{z}=2.3_{-0.3}^{+0.1}$ and $k T_{z}=2.2 \pm 0.2$. Given that $k T_{z}$ is always slightly lower than or consistent with $k T_{\mathrm{e}}$, we conclude that there is no indication of overionization in the central region of W49B.

\section{Discussion}

On the basis of low angular-resolution $A S C A$ line images, Fujimoto et al. (1995) argued for stratification of the SN ejecta in W49B, with iron confined to the inner region and lower- $Z$ elements ( $\mathrm{Si}$ and $\mathrm{S}$ ) forming an outer envelope. This hypothesis was supported by the analysis of the global spectrum, with the intensity ratios of $\mathrm{Ly} \alpha$ and $\mathrm{He} \alpha$ giving different ionization ages for $\mathrm{Si}$, 
S, Ar, and Ca. Hwang et al. (2000) pointed out that, while this conclusion is compatible with the ASCA spectrum, no difference in the ionization ages is required if the data are modeled with a two-component thermal plasma. The analysis of XMM-Newton data allowed us to discriminate between these two scenarios. Our spatially resolved spectral analysis has shown that a correct fit of the spectra requires two thermal components and that these two components are compatible with collisional ionization equilibrium, both at the center and in the outer parts of the remnant. Moreover, as explained in Sect. 3.2, our line images and equivalent width maps exclude a radial stratification of the elements in the ejecta, with Fe K emission confined to the inner part of the X-ray emitting region and lower-Z emission lines originating in an outer shell. In fact, both the central barrel-shaped structure and the eastern elongated structure of the SNR have high abundances of $\mathrm{Ar}, \mathrm{Ca}$ and $\mathrm{Fe}$, while the western part has lower abundances.

The physical conditions of the plasma are not uniform in W49B. The highest temperatures are found in the northern end of the elongated eastern structure (labelled $a$ ) and in the central barrel-shaped structure (labelled $b$ ). The lowest temperatures are found in the western part of W49B and in the south eastern zone. Assuming pressure equilibrium between the two components, no significant variations in the plasma density across the remnant are observed. The observed X-ray emission originates in the SN ejecta, and $\mathrm{Si}, \mathrm{S}, \mathrm{Ar}, \mathrm{Ca}, \mathrm{Fe}$, and $\mathrm{Ni}$ are all highly overabundant with respect to their solar values. They have almost the same values in structures $a$ and $b$, and are lower (but still overabundant) in structure $c$. No clear evidence of X-ray emission from the shocked ambient medium is present. In fact, the results of the spatially resolved spectral analysis suggest that both the X-ray emitting components are associated with overabundant plasma. This agrees with the similarity of the morphology between the Si He-like emission blend (associated with the cooler component) and the other emission blends (associated with the hotter component). As explained in Sect. 3.3, however, the high absorption column inhibits the possibility of a detailed study of the soft component in that it is not easy to determine its morphology. Furthermore, the contribution of the hot component is significant even below $1.7 \mathrm{keV}$, so that it is not possible to produce a clean image of the soft component. We therefore cannot rule out the possibility that the soft component may be associated with the shocked circumstellar or interstellar medium.

It is interesting to consider to what extent the complex morphology of W49B, and the inhomogeneities in temperature and abundance, are caused by the dynamics of the SN explosion or by the remnant's interaction with its environment. Recent 2.12 micron infrared imaging observations have revealed a shocked molecular $\mathrm{H}_{2}$ cloud that confines the remnant (Fig. 2). The circular distribution of the molecular cloud around the SNR has probably been shaped by strong stellar winds from the progenitor star pushing out the ambient interstellar material to its current location and forming a cavity. Infrared [Fe II] observations at $1.64 \mu \mathrm{m}$ reveal a barrel-shaped structure with four coaxial rings of warm gas, which has been interpreted as residuals of a strong stellar wind (Keohane et al. 2004).

The morphology of the eastern region of W49B has clearly been shaped by the ambient molecular cloud. Despite their similar abundance values, the eastern $a$ and central $b$ regions have very different morphologies. The fact that region $a$ is almost perpendicular to region $b$ is probably due to its confinement by the large $\mathrm{H}_{2}$ cloud to the east. The [Fe II] observations show that the cavity is an inhomogeneous environment for the supernova remnant expansion.
In contrast, there are no observed interstellar structures that might have shaped the elongated structure of the central X-ray barrel $b$. This centrally barrel-shaped structure thus may have been produced directly by the supernova explosion or by preexisting structures in the ambient stellar wind material.

The central barrel morphology seen in the Chandra Fe K image has been attributed to a bipolar jet and taken as evidence that $\mathrm{W} 49 \mathrm{~B}$ is a $\gamma$-ray burst (GRB) remnant ${ }^{1}$. The presence of such an elongated morphology, however, does not necessarily reflect a jet-like explosion. A similar elongated central bright structure has been observed with Chandra in the G292.08+1.8 remnant, but Park et al. (2004) showed that its emission is associated with normal chemical composition plasma that originates in shocked dense circumstellar material rather than in shocked ejecta. Unlike for G292.08+1.8 SNR, our analysis suggests that the X-ray emission in the central X-ray barrel of W49B is dominated by ejecta, although we cannot rule out the possibility that the soft component comes from solar abundance material. The scenario proposed for G292.08+1.8 can nevertheless be invoked for W49B. It is possible that the barrel-like X-ray emission reflects the morphology of the ambient medium across the remnant. While no dense structures are visible toward the North and South of W49B (as shown in the infrared image of Fig. 2), we may assume the ambient medium to be enhanced in the central zone, forming a torus-like structure all across W49B. According to this scenario, the cold component may be associated with the thin belt of material heated by the transmitted shock travelling into the ISM structure. This would explain the low values of the filling factor of the soft component. The hot component is associated with the ejecta interacting with the reflected shock. In this case, only the material expelled in the plane of the torus is interacting with the reverse shock and heated by it. Notice, however, that according to our estimates of the plasma density for the spectral regions, the total mass of the ejecta in the hot component in these regions is $\sim 8 M_{\odot}$. This indicates that, if we assume that we are observing only a small fraction of the ejecta, we probably overestimate the total expelled mass.

Another possibility is that the barrel is associated with a bipolar ejecta jet interacting with the cavity wall visible at the eastern end of the remnant. In this case we have to assume that the density contrast has driven a very strong reverse shock that has now reached the center of W49B. Moreover, we have found that structures $a$ and $b$ have similar abundance values and, therefore, that $a$ could be considered as the head of the eastern jet. The scenario that emerges is that this jet has been distorted to deviate mostly southwards by its impact with the $\mathrm{H}_{2}$ wall. In this framework, we would have a highly anisotropic bipolar jet, where the eastern arm is significantly more extended, hot and Fe-rich than the western one.

Jet-like explosions have been investigated as possible mechanisms for $\gamma$-ray bursts (GRB), because a spherically symmetric GRB would require an extremely high energy (several times the solar rest mass). A link between very energetic SNe and GRBs has been found by Galama et al. (1998), who showed that the bright type Ic SN 1998bw (with an estimated energy $E \sim 3 \times 10^{52} \mathrm{erg}$, Iwamoto et al. 1998) is the probable optical counterpart of the $\gamma$-ray burst GRB 980425 . Observations of SN 1998bw have shown strong indications of asphericity in the explosion (Mazzali et al. 2001). Indications of aspherical explosions have also been found in many other hypernovae, i.e.

\footnotetext{
1 http://chandra.harvard.edu/press/04_releases/ press_060204.html
} 


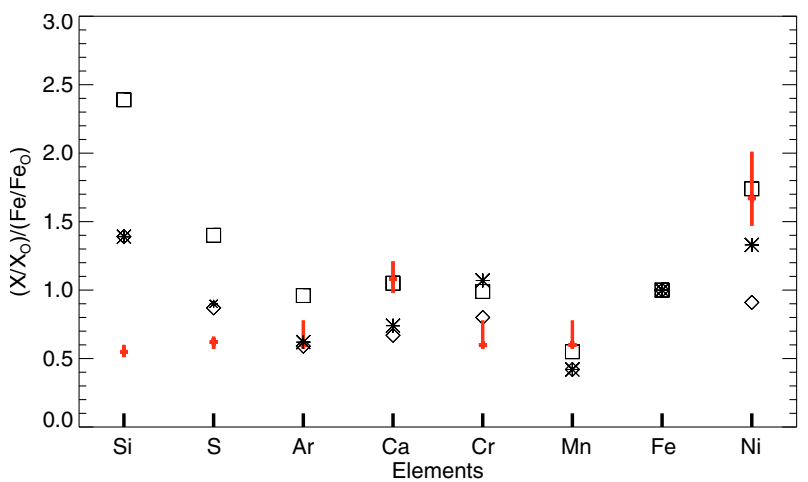

Fig. 8. Elemental abundances in the central X-ray barrel of W49B (red crosses, see Table 4), normalized to the $\mathrm{Fe}$ abundance relative to the solar values (Anders \& Grevesse 1989), compared with the yields obtained from the aspherical explosion models 25a (boxes) and 25b (diamonds) and from the spherical model $25 \mathrm{Sa}$ (stars) of explosive nucleosynthesis by Maeda \& Nomoto (2003). The $\mathrm{Cr}$ and $\mathrm{Mn}$ abundances in the central barrel have been derived from the Ar abundance following Hwang et al. (2000).

supernova explosions with $E \gtrsim 10^{52} \mathrm{erg}$ (for a review on hypernovae see Nomoto et al. 2003).

The relationship between asymmetric hypernovae and GRB agrees with the collapsar model (MacFadyen \& Woosley 1999), in which the collapse of a massive rotating core can generate a black hole and supersonic jets that propagate through the star. The nebular spectrum of SN 1998bw is reproduced by such a model well. Khokhlov et al. (1999) have shown that this mechanism produces a highly aspherical explosion with bipolar fast jets. A bipolar explosion does not necessarily imply a GRB, however, because a high explosion energy $\left(\gtrsim 10^{52} \mathrm{erg}\right)$ is also required.

To investigate the possibility that W49B is a GRB remnant, we compared the abundance values found in the $a$ barrel (see Table 4) with those obtained from models of explosive nucleosynthesis in bipolar explosions by Maeda \& Nomoto (2003). In particular, we compared the expected and observed ratios $\left(X / X_{\odot}\right) /\left(\mathrm{Fe} / \mathrm{Fe}_{\odot}\right)$ for $X=\mathrm{Si}, \mathrm{S}, \mathrm{Ar}, \mathrm{Ca}, \mathrm{Cr}, \mathrm{Mn}$, and $\mathrm{Ni}$ (for $\mathrm{Cr}$ and $\mathrm{Mn}$ we assumed the same ratios as observed for Ar, in agreement with the estimates given in Hwang et al. 2000). None of the models can reproduce the entire observed abundance pattern. The hypernova models with zero-age main-sequence progenitor mass $M_{\text {ZAMS }}=40 M_{\odot}$ and explosion energy $E>10^{52} \mathrm{erg}$ strongly disagree with the observed values (with very large discrepancies for the $\mathrm{Si}, \mathrm{S}$, and $\mathrm{Ar}$ abundances). We obtained better results with the less energetic models $25 \mathrm{~A}\left(E=6.7 \times 10^{51}\right.$ and $\left.M_{\text {ZAMS }}=25 M_{\odot}\right)$ and $25 \mathrm{~B}\left(E=0.6 \times 10^{51}\right.$ and $\left.M_{\text {ZAMS }}=25 M_{\odot}\right)$. We also found relatively good agreement with the spherical explosion model 25Sa $\left(E=1 \times 10^{51}\right.$ and $\left.M_{\text {ZAMS }}=25 M_{\odot}\right)$. The predicted values of these three models and our observational findings are summarized in Fig. 8. There is good overall agreement for Ar through $\mathrm{Ni}$, but the observed abundances of $\mathrm{Si}$ and $\mathrm{S}$ are not well reproduced.

While the observed abundance pattern in W49B does not favor a hypernova explosion of sufficient energy to generate a GRB, we cannot definitely exclude the possibility that W49B was related to such an event.

\section{Summary and conclusions}

Our study of the XMM-Newton observations of the Galactic supernova remnant $\mathrm{W} 49 \mathrm{~B}$ has shown that:

- The remnant presents a complex X-ray morphology characterized by centrally barrel-shaped emission $(b)$, terminated on the eastern side by an elongated perpendicular structure $(a)$, and on the western side by a more diffuse, nearly aligned structure $(c)$.

- The physical conditions of the plasma are not homogeneous throughout the remnant. Lower temperatures are found in the remnant's western end, while hotter regions are located in the center and northeast.

- A high $\mathrm{Ni}$ overabundance $\left(\mathrm{Ni} / \mathrm{Ni}_{\odot}=10_{-1}^{+2}\right)$ was measured in the central barrel, and the presence of $\mathrm{Cr}$ and $\mathrm{Mn}$ lines in the global spectrum is confirmed.

- We found no evidence of overionization of the plasma in the center of W49B.

- The X-ray emission from all the observed regions of W49B originates in the ejecta, based on significant element overabundances of $\mathrm{Si}, \mathrm{S}, \mathrm{Ar}, \mathrm{Ca}$, and $\mathrm{Fe}$. The equivalent-width maps of W49B and a detailed spectral analysis of individual regions both show that the central $(b)$ and eastern $(a)$ structures have similar abundances, and the western $(c)$ region exhibits lower abundances. This decrease is particularly pronounced for iron.

- The X-ray morphology of W49B may be attributed to preexisting structures in the ambient medium, which may have produced an aspherical reverse shock, or, alternatively, to an aspherical supernova explosion in a wind cavity (bounded by molecular clouds on the eastern side of the remnant). Because it has abundances similar to those in the central barrel, the eastern structure $a$ can be considered as the head of the jet. It is distorted and deviates mostly southward due to its impact with the dense molecular wall that confines its expansion to the east.

- We investigated the possibility that W49B is a $\gamma$-ray burst remnant by comparing the observed abundances with yields derived from supernova and hypernova nucleosynthesis models. We found better agreement with the less energetic $\left(E \sim 10^{51} \mathrm{erg}\right)$ models. The association of W49B with a $\mathrm{GRB}$ is therefore not necessarily implied by the spectral data.

Acknowledgements. We thank the referee, Dr. B. Aschenbach, for his comments and suggestions. The authors wish to thank Joseph Lazio for providing us with the $327 \mathrm{MHz}$ radio image of W49B. M.M. thanks Costanza Argiroffi for the discussions about the ionization temperature. This work was partially supported by the Ministère Français des Affaires Etrangères and by the Ministero dell'Istruzione, dell'Università e della Ricerca - Rif. Prog. N. 78.

\section{References}

Anders, E., \& Grevesse, N. 1989, Geochim. Cosmochim. Acta, 53, 197 Arnaud, K. A. 1996, in Astronomical Data Analysis Software and Systems V, ASP Conf. Ser., 101, 17

Arnaud, M., Neumann, D. M., Aghanim, N., et al. 2001, A\&A, 365, L80

Bleeker, J. A. M., Willingale, R., van der Heyden, K., et al. 2001, A\&A, 365, L225

Bocchino, F., Maggio, A., \& Sciortino, S. 1999, A\&A, 342, 839

Fujimoto, R., Tanaka, Y., Inoue, H., et al. 1995, PASJ, 47, L31

Galama, T. J., Vreeswijk, P. M., van Paradijs, J., et al. 1998, Nature, 395, 670

Hwang, U., Petre, R., \& Hughes, J. P. 2000, ApJ, 532, 970 
Iwamoto, K., Mazzali, P. A., Nomoto, K., et al. 1998, Nature, 395, 672 Kawasaki, M., Ozaki, M., Nagase, F., Inoue, H., \& Petre, R. 2005, ApJ, 631, 935 Keohane, J. W., Reach, W. T., Rho, J., \& Jarrett, T. H. 2004, Amer. Astron. Soc. Meet. Abstr., 204

Khokhlov, A. M., Höflich, P. A., Oran, E. S., et al. 1999, ApJ, 524, L107 Lacey, C. K., Lazio, T. J. W., Kassim, N. E., et al. 2001, ApJ, 559, 954 Lampton, M., Margon, B., \& Bowyer, S. 1976, ApJ, 208, 177 Liedahl, D. A., Osterheld, A. L., \& Goldstein, W. H. 1995, ApJ, 438, L115 MacFadyen, A. I., \& Woosley, S. E. 1999, ApJ, 524, 262

Maeda, K., \& Nomoto, K. 2003, ApJ, 598, 1163

Mazzali, P. A., Nomoto, K., Patat, F., \& Maeda, K. 2001, ApJ, 559, 1047

Mewe, R., Gronenschild, E. H. B. M., \& van den Oord, G. H. J. 1985, A\&AS, 62, 197

Mewe, R., Lemen, J. R., \& van den Oord, G. H. J. 1986, A\&AS, 65, 511 Moffett, D. A., \& Reynolds, S. P. 1994, ApJ, 437, 705
Nomoto, K., Maeda, K., Umeda, H., et al. 2003, in A Massive Star Odissey, from Main Sequence to Supernova, ed. K. A. van der Hucht, A. Herrero, \& C. Esteban (ASP), Proc. IAU Symp., 212, 395

Park, S., Hughes, J. P., Slane, P. O., et al. 2004, ApJ, 602, L33

Pye, J. P., Thomas, N., Becker, R. H., \& Seward, F. D. 1984, MNRAS, 207, 649 Radhakrishnan, V., Goss, W. M., Murray, J. D., \& Brooks, J. W. 1972, ApJS, 24, 49

Read, A. M., \& Ponman, T. J. 2003, A\&A, 409, 395

Rho, J., \& Petre, R. 1998, ApJ, 503, L167

Smith, A., Peacock, A., Jones, L. R., \& Pye, J. P. 1985, ApJ, 296, 469 Strüder, L., Briel, U., Dennerl, K., et al. 2001, A\&A, 365, L18

Turner, M. J. L., Abbey, A., Arnaud, M., et al. 2001, A\&A, 365, L27

Willingale, R., Bleeker, J. A. M., van der Heyden, K. J., Kaastra, J. S., \& Vink, J. 2002, A\&A, 381, 1039 